\title{
Multidisciplinary pediatric brain tumor clinics: the key to successful treatment?
}

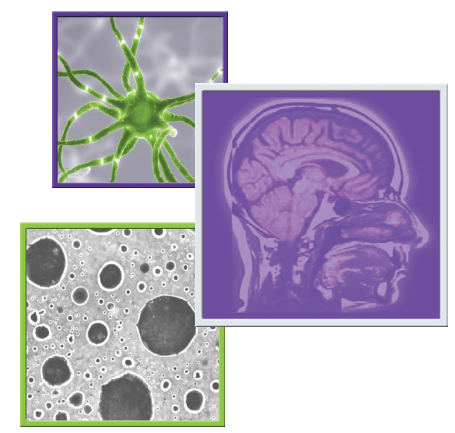

Mohamed S Abdel-Baki ${ }^{* \neq, 1}$, Emily Hanzlik ${ }^{\ddagger, 1}$ \& Mark W Kieran ${ }^{\ddagger, 2}$

\section{Practice points}

- As diagnosis and treatment of pediatric brain tumors continues to advance, a continuously increasing number of healthcare providers are involved in the management and follow-up of these patients.

- Multidisciplinary clinics have become the standard of care for cancer patients, including those with brain tumors. Multidisciplinary pediatric cancer centers are present throughout the USA, but with limited guidelines on the necessary components that are specific to brain tumor patients.

- The American Academy of Pediatrics has recently updated and published their recommendations regarding the faculty, facilities and technology that should be present in centers that treat pediatric oncology patients.

- Multidisciplinary brain tumor clinics require support from the institution and the hospital as well as having multiple specialties including neurosurgery, oncology, neurology and radiation oncology. Other specialties should be available for consult and referrals in regard to long-term effects of both the tumor itself and effects of treatment.

- Multidisciplinary brain tumor clinics should have an established meeting time that all faculty and personnel involved can attend to ensure optimal patient care. A designated provider should be in charge of presenting patients at these meetings and ensuring that the family is involved in discussions.

- Within the multidisciplinary clinics, patients should have access to community support groups, family support and counseling, and school support to help with reassimilation into school and society as well as to assess progress in the years following diagnosis and treatment.

- Barriers to successful multidisciplinary clinics are known to exist at both institutional and faculty levels. Recognizing the barriers and establishing ways to address concerns are essential to a successful multidisciplinary brain tumor clinic.

SUMMARY Tumors of the CNS are the most common solid tumors diagnosed in childhood. As technology and research in cancer care are advancing, more specialties are involved in the diagnosis, treatment and follow-up of children with brain tumors. Multidisciplinary clinics have become the standard of care for cancer care throughout the USA, and specialty clinics focused on particular cancer types are gaining attention in improving the patient outcomes and satisfaction. We will discuss the role of multidisciplinary clinics, in an attempt to create preliminary guidelines on establishing and maintaining a multidisciplinary brain tumor clinic in order to optimize the care of the patients and their families.

'Cardinal Glennon Children's Medical Center, St Louis University School of Medicine, 1465 S. Grand Boulevard, St Louis, 


\section{KEYWORDS}

- brain tumors $\bullet$ long-term outcomes $\bullet$ multidisciplinary

- neuro-oncology $\bullet$ pediatric brain tumor clinic $\bullet$ quality of life $\bullet$ tumor boards
Tumors of the CNS are the second most common type of pediatric cancer and the most common type of solid tumors seen in children, accounting for almost $20 \%$ of all malignancies in children aged $0-18$ years [1]. With the advancements in diagnosis and treatment of these tumors, the survival rate has continued to increase and now approaches $80 \%$. Of those diagnosed with the two most common types of brain tumors, pilocytic astrocytoma and medulloblastoma, survival rates have increased from 81.1 to $93.0 \%$ and 43.7 to $62.8 \%$, respectively, since the 1980 s. Increased survival is multifactorial and in part can be attributed to the advancements in imaging, new and improved neurosurgical techniques and new therapeutic modalities [2]. Increasing evidence suggests that a significant aspect of this improvement in outcome for children with tumors of the CNS is related to integrated care between specialties.

With the increase in number of diagnosed brain tumors each year along with the continued improvement in survival, there are more children living with brain tumors or are long-term survivors, many of whom will have significant sequelae from their tumor and its treatment. For all brain tumor patients, optimal care is essential to improved survival and quality of life. Pediatric cancer centers and specifically brain tumor clinics have become the standard of care across the nation in order to optimize the care for pediatric brain tumor patients and their families. Multidisciplinary clinics have been used in many complex medical conditions including other types of cancers and chronic medical conditions. With the emergence of multidisciplinary clinics in the care of pediatric brain tumors, questions arise about the keys to success of these clinics and the long-term outcomes of the patients that come through these clinics during the course of their illness.

\section{Multidisciplinary approach in pediatric cancer centers}

Multidisciplinary care can be described with varying terms including multidisciplinary teams, multidisciplinary care conferences and tumor boards. A multidisciplinary approach to healthcare involves a wide range of health professionals who can each make unique contributions to patient care and improving outcomes. It also includes a forum for the individuals involved to meet at specific times to discuss patient management going forward [3,4]. Multidisciplinary cancer care has become the gold standard in the USA for a majority of cancer types in both the adult and pediatric populations and have been an established part of complete cancer care for more than 50 years. The Commission on Cancer and the American College of Surgeons both require that multidisciplinary teams be in place in healthcare centers accredited with delivering care to cancer patients [3]. Multidisciplinary teams for the care of cancer patients were most well established nationwide and internationally initially in the breast cancer population. They began as a way to address the evolving treatment by a variety of new modalities for optimal management and treatment, as more specialists were required to assist in the overall care of these patients [4]. Although multidisciplinary cancer teams and tumor boards have become the standard of care, there is little evidence-based medicine on the benefits of outcomes for patients involved. Comprehensive treatment and decision making by specialists, improved patient satisfaction, ongoing education for healthcare providers and increased access to clinical trials are all proposed benefits of multidisciplinary clinics [5]. There is also evidence that tumor board recommendations are often carried out by the team for the benefit of the patient and can play a tactical role in their care [6]. The difficulty in studying outcomes of multidisciplinary centers is complicated by the nature of the complexity of the disease as well as the lack of ability to now perform randomized control trials to prove that multidisciplinary clinics are the standard of care and do improve the outcomes. Furthermore, there has been limited standardization of tumor boards, definitions and components of what makes up an effective multidisciplinary team vary between centers. A review by Hong et al. from 2010 looking at the relationship between survival and multidisciplinary teams identified 12 studies that showed significant improvement in survival of patients that participated in multidisciplinary cancer clinics across several different types of cancers. However, the interpretation of the data was limited because of methodological heterogeneity of the studies as well as the retrospective and 'before and after' types of studies that were included [5].

As multidisciplinary cancer clinics are becoming the gold standard, the American Academy of Pediatrics has established Standards for Pediatric Cancer Centers that would apply to any pediatric brain tumor clinic established within these 
centers. Each center must have certain facilities available to be qualified to treat complex pediatric cancer patients (Box 1) [7]. There are limited guidelines published about the development of a brain tumor multidisciplinary clinic and how they should be run. In 2014, the European Council proposed a policy statement that reflected the existing expert opinion on multidisciplinary care for brain tumors and suggested the following objectives:

- Care objectives: early access to the clinic based on preoperative assessment and pathology, with the decision-making process based on evidence-based medicine and established protocols. Treatment should be discussed with the patient throughout the process with routine surveillance for relapse and post-treatment needs;

- Organization objectives: every new case should be presented at tumor board and the team should monitor all recurrent and new cancer patients. There should be a chair to coordinate patient lists to be presented, monitor attendance at meetings and implement decisions made by the team;

- Clinical assessment: data about the processes including time to diagnosis, time to first treatment and relapse rates should be analyzed periodically to identify factors that can be improved upon;
- Patient's rights: patients should be able to identify a responsible physician at each stage of their care process and all treatment preferences should be discussed with patient before clinical decisions are made. Patients should be provided with community resources, support groups, and supplement information [8].

Overall, there should be a flow to the workings of a multidisciplinary team involving the exchange of all the information and patients among the members of the team, presentation of therapeutic options, obtaining patient's input on the various discussed methods and determining an individual treatment plan with the patient [9]. These suggestions for multidisciplinary clinics are the backbone and can be applied to any multidisciplinary cancer clinic including pediatric brain tumor clinics.

\section{Brain tumor clinics - multidisciplinary approach}

The ideal way to organize and run multidisciplinary clinics depends on the type of patients being treated, but there are standards of care that are now being established to maximize the efficiency and benefit of such clinics. Establishing a pediatric brain tumor clinic requires the involvement of many specialties that overlap with those required for other multidisciplinary cancer centers as well as unique aspects of care specific to

Box 1. Facilities and capabilities required for pediatric cancer centers.

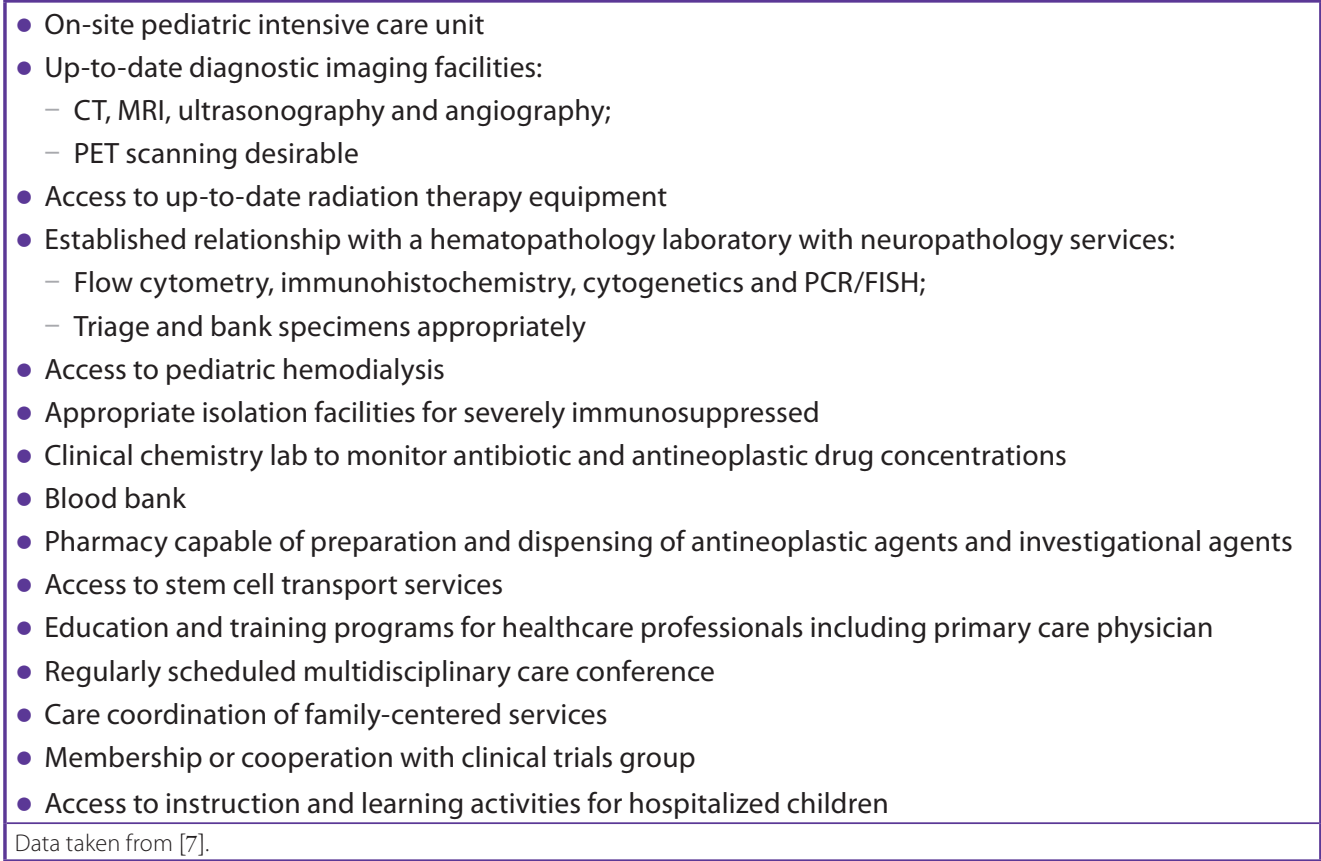


pediatrics and brain tumor patients. It has been established since the 1970s that pediatric brain tumor patients have higher cure rates with the fewest sequelae when they are diagnosed and treated in locations with pediatric specialists and advanced technology. Pediatric patients treated at cancer centers had fewer surgical mortalities and lower 3-year relapse rates as compared with those treated at noncancer centers [10]. At cancer centers, patients with medulloblastoma were more likely to be diagnosed earlier due to better technology and were treated on established protocols which resulted in better outcomes [10]. It has also been found that in adult brain tumor patients, multidisciplinary clinics improved patient outcomes by coordination of patient care, adherence to established clinical protocols and team communication. In a randomized control trial of adults with glioblastomas, there was a significant increase in the number of patients who underwent repeated imaging, began radiation therapy sooner (within 28 days) and received adjuvant chemotherapy if treated in a multidisciplinary clinic versus a traditional on-call referral program [11]. In this population, there was evidence that delay in beginning radiation therapy was associated with decreased survival suggesting that the reduction in number of days to treatment of those enrolled at a multidisciplinary clinic would be expected to lead to increased survival [12]. There is also evidence that the outcomes in pediatric brain tumor resections are better in hospitals with a higher volume of cases and where increased numbers of pediatric specialties are available [13]. Over time, the number of procedures on brain tumor patients being done at teaching hospitals and the number performed by pediatric specialists has increased and resulted in lower mortality and better hospital discharge dispositions [13]. This suggests that for pediatric brain tumor patients, care at facilities that are specialized in pediatric oncology and neurosurgery seem to have better outcomes.

In developing brain tumor clinics, in addition to following the guidelines for generic multidisciplinary clinics as well as meeting the personnel and facility requirements of pediatric cancer centers, aspects must be addressed related specifically to brain tumor patients. Leadership of the clinics must include, in addition to support from the institution and the departments involved, a pediatric neurosurgeon, radiation oncologist and a physician dedicated to neurooncology. For those with a high disease burden or mass effect and the need for pathological diagnosis, surgical intervention is an essential first-line therapy. Radiation therapy is a crucial element of treatment and the involvement of a radiation oncologist ensures that the choice of radiation therapy modality is thoroughly investigated and carried out. Finally, following surgery and radiation therapy, chemotherapy becomes an integral part of therapy for many brain tumor patients. Neuro-oncologists are essential to determine the treatment protocol and often become the most used point of access for patients as they move along through their therapies [14] . Other departments involved in the brain tumor clinic should include: neuroradiology, and neuropathology. The board should also consist of a coordinator whose job will be to prepare the patient list for the meetings and ensure that the plans are followed through after decisions have been made as well as coordinate the care among the multiple services involved. Another important component is a database that is accessible to each party involved and has a methodology for updating information that is accessible to all participants. Other specialities that are usually involved, include audiologists, ophthalmologists, endocrinologists and a variety of other specialties tailored to each child's particular needs. Often overlooked in the development of multidisciplinary clinics are the ancillary staff, including social workers, psychologists and back to school specialists. Meetings should occur at regularly scheduled intervals, and the ability to visually present both images and pathology should be available in the meeting space [15]. Each aspect of care for the patients and their families should be available including diagnosis and treatment stages, long-term follow-up of survivors, caregiver support and end of life/palliative care.

\section{- Initial diagnosis \& treatment}

During the initial diagnosis and treatment of pediatric brain tumors, parents and patients are presented with shocking news, a large amount of information, and short time frame to process information and make decisions. Parents and patients quickly become overwhelmed with the information and brain tumor clinics should use this opportunity to maximize the contact with the patient to ensure understanding. Brain tumor clinics provide the opportunity for all specialties to evaluate the patient together and create the best treatment plan. The importance of speed in the decision-making process is often monumental 
in favorable outcomes. The ability to come to an agreement and implement therapy is an important benefit of brain tumor clinics. It provides a basis for educational discussion of all angles of treatment options as well as providing the patient with all aspects of care without forcing the family to search for each specialty separately and potentially end up with care at several different locations [16]. For parents, learning to navigate the healthcare system when their child is diagnosed is a daunting task that can be simplified with the multidisciplinary approach. Physicians are cited as the most trusted source of information for the patient and family [16,17]. Availability to all physicians involved in the care at one time and location allows patients and families to assimilate information, encourages cooperation among different specialties and ensures follow through on the decisions. Multidisciplinary clinics are also an efficient use of time for the patient. In a parental survey, parents felt that social functioning and independence for their children were the most important aspects of quality of life when living with a brain tumor [18]. Multidisciplinary brain tumor clinics ensure that patients' time is well spent and that multiple needs can be addressed in one visit. This enables more regular attendance at school and social events, which are important factors in quality of life during the diagnosis and treatment phase when frequent visits to the hospital are required. Multidisciplinary clinics address many of the concerns of both physicians and parents during the diagnosis and treatment phase of brain tumor management.

\section{- Survival \& long-term follow-up}

An important component essential to brain tumor clinics is the continued follow-up of patients to both assess recurrence and long-term sequelae of survivors. As survival rates continue to improve, more and more children will be living into adolescence and adulthood with continued symptoms of the brain tumor or as a consequence of the treatment. Eighty percent of children with a history of a brain tumor will experience some type of long-term morbidity [19]. Multidisciplinary clinics must be prepared and equipped to deal with the ongoing physical needs of survivors. In regard to long-term physical health sequelae, brain tumor survivors are more affected than other pediatric cancer survivors. McCartney et al. found that there is a medium of six symptoms per patient in brain tumor survivors, with the most common and most troublesome being lack of energy, sleep disturbances and headaches [20]. Known consequences of brain tumors and the treatment include hearing loss, learning difficulties, balance issues, headaches and seizures that will affect the quality of life of the child and will require continued care within the brain tumor clinic [21]. Neurocognitive sequelae are potentially the most prominent late effects in brain tumor survivors as a result of the tumor, surgical resection and the effects of radiation/chemotherapy including decline in IQ along with difficulties in attention, memory and processing speed [22]. Endocrine abnormalities including hypothyroidism, growth hormone deficiency and delayed puberty were found in $43 \%$ of childhood brain tumor survivors. They were most likely to be seen in those with optic pathway and hypothalamic tumors and in those treated with whole brain irradiation. Growth hormone was found to be deficient in $100 \%$ of patients who received greater than 36 Gy to the pituitary region, and hypothyroidism was seen in the majority of patients receiving any amount of radiation [23,24] . Cardiovascular complications including stroke, blood clots and angina were found in $18 \%$ of survivors [23]. Psychiatric problems are also seen in brain tumor survivors including maladjustment, depression, anxiety, personality issues and psychosis. Adolescents particularly are at high risk because they are entering a time where they are expected to function at a more independent level with increasing workloads. However, this is the time period in which late effects of the tumor or therapy may be emerging [24]. Suicidal ideations have also been shown to be increased in young adult survivors as compared with the general population. Among survivors, those with a history of seizures as well as those treated with surgery only were associated with higher rates of suicidal ideations [25]. In multidisciplinary brain tumor clinics, it is prudent to have a screening tool to identify psychological problems, especially depression, in order to identity those who would benefit from psychiatric referral. The Beck Depression Inventory for Youth is a wellvalidated screening tool for identifying patients at risk. Blackmon et al. found that many items on the 20-point screen are correlated and recommended using a shortened eight-item form with a cutoff score of greater than or equal to five in adolescent cancer survivors. The shortened screening tool would be ideal in brain tumor clinics where time may be limited, but could still be effective in identifying the patients in need of further 
evaluation [26]. While not necessarily required as part of the multidisciplinary brain tumor clinic's regular meetings, specialists including endocrinologists, cardiologists, psychiatry, counseling and sleep medicine need to be involved in the care of these patients and easily accessible for referral from brain tumor clinic providers.

Quality of life is an important aspect of care that brain tumor clinics should be prepared and equipped to address with patients as a multidisciplinary entity at each clinic visit. Patients have shown to not only have worsened physical health but also worsened psychological, emotional and social health with decreased performance at school [27]. There are screening tools available to assess quality of life in children outside of the physical domain. The PedsQL 4.0 is age specific and a rapid assessment that can be performed at scheduled visits and has been validated as a measurement of quality of life in brain tumor survivors [27]. In addition to screening and recognizing factors affecting the quality of life in these survivors, it is important for brain tumor clinics to have access to resources to address each facet that is affecting an individual's quality of life. Quality of life is multifaceted and addressing each component for the children is essential to ensuring their ability to function in society [28]. Social workers, school interventions, neuropsychological testing, community support groups and counseling should be resources the family is made aware of through the clinic, especially as children make the transition from the acute stage of the illness to remission and reassimilation into school and society [17].

\section{- Caregiver support}

Another aspect of a successful brain tumor clinic that affects quality of life is awareness of the parental/caregiver burden and the ability to address these aspects throughout the diagnosis, treatment and follow-up. Parents of survivors have increased levels of post-traumatic stress disorder and general psychological distress [29]. Caregivers also report poorer physical and emotional health with increased career sacrifices, workplace discrimination and neglect of other family members, including other children [17]. Studies have shown that stress and psychological problems in the parents were found to correlate with decreased cognitive functioning and poorer quality of life in multiple domains for the child including school, social and emotional subsets of quality of life [30-32]. Brain tumor clinics should address the caregiver's needs in addition to the child's to ensure optimized quality of life. Therefore, health education done by physicians, nurses and social workers should be included in the multidisciplinary approach. In addition, a social worker or personnel specific to oncology can help bridge gaps between healthcare and insurance companies to ensure that services and equipment needs are met to lessen the burden on caregivers [17]. Access to community resources not only for the child but also for the family and other siblings including both parental and family counseling should be established through the clinic coordinator and ancillary staff who are available at scheduled clinic visits to address issues and make appropriate referrals. This whole approach to multidisciplinary brain tumor clinics ensures that all aspects of care that can affect the outcomes and functioning of the patients are met and attended to, organized in a central location.

\section{- Palliative care}

While survival rates of childhood CNS tumors are increasing and nearing $80 \%$, cancer is still the leading cause of disease-related death in children [1]. An important component of multidisciplinary brain tumor clinics is palliative care with pain management, hospice services and bereavement counseling. The American Academy of Pediatrics recommends that palliative care be offered at the time of diagnosis and continue through the illness course regardless of the outcomes [33]. A survey of 187 institutions in the Children's Oncology Group found only 58\% of the institutions had a palliative care service. Of those institutions, only a median of three patients of the 45 newly diagnosed cancer patients were using the palliative care team [34]. Only one-third of children who subsequently died of cancer were referred to palliative care services [35]. Palliative care services continue to be inadequately available and underused. In multidisciplinary brain tumors clinics, where end-of-life issues arise in up to $20 \%$ of its patients, palliative care teams with a focus on pain and symptom management should be available from the time of diagnosis. Referral to specialist pediatric palliative care in cancer patients has been shown to be significantly associated with both decreased planned and emergency hospital admissions, which can potentially be of personal and social benefit to a child nearing the end of life [35]. Brain tumor clinics can establish early relationships between palliative care and the patients in order 
to optimize their care and comfort. Identifying those patients who are in significant pain and in need of pain management services can be difficult during clinic visits. There are many pain scales available but are often multifactorial with many questions that can be long and difficult for children, especially those with neurocognitive deficits. Chordas et al., in an attempt to find the most useful scale while still providing sufficient sensitivity and specificity, found that the pain thermometer, a single factor pain scale, was not sensitive enough to pick up patients found to have significant pain scores on other multifactorial questionnaires. They did, however, use a 15-item Brief Pain Survey, which is shorter and simpler than the Brief Pain Inventory. The Brief Pain Survey is more appropriate in the younger patients and those with neurocognitive deficits. Although a single factor pain scale did not reach appropriate sensitivity to recognize those in significant pain, an abbreviated multifactorial questionnaire can still be used in brain tumor clinics to assess the status of the patients and guide further management [36].

\section{- Barriers to successful multidisciplinary clinics}

Limitations to effective teamwork

Surveys have shown that effective teamwork and communication are essential to the success of multidisciplinary clinics. First, for an effective team to work, there must be institutional support for the clinic including financial, time, and resources made available to the team to optimize the meetings and access to patients [37]. Other concerns that affect teamwork include time pressures, low attendance by members of the team, lack of leadership and excessive caseload [4,37,38]. Regarding time constraints, most felt that the assigned time for meetings was insufficient for full discussion and agreement on treatment protocols. This led to patients either being rushed through or pushed to the next meeting, delaying patient care. Low attendance by key team members also limited the ability to have a full discussion about each patient and led to inappropriate treatment plans [38].

\section{Implementation of treatment decisions}

Whether the treatments decided on by the multidisciplinary team are implemented is often considered a measure of the success and was found to be implemented $85-95 \%$ of the time. The most common reasons for decisions not being implemented were lack of information available including patient preference and limited close contact with the patient, insufficient evidence of patient comorbidities and interval disease progression [37,38]. Ensuring all information is collected prior to meetings including tests and image results would reduce the impact of these barriers. Also, ensuring that the patient's wishes have been discussed up front can save valuable time when considering the options among the multidisciplinary team.

\section{Cost-effectiveness}

Multidisciplinary clinics can be resource intensive including increased time for the physicians and personnel involved, access to adequate meeting spaces and exam rooms, and technology including databases and ways to share information among team members [4]. A recent systematic review that looked at 15 studies evaluating the cost effectiveness of multiple different types of multidisciplinary clinics found insufficient evidence to draw any conclusions about their overall financial burden on institutions [39].

\section{Conclusion \& future perspective}

Multidisciplinary brain tumor clinics within pediatric cancer centers have become the standard of care despite limited evidence-based data of their success on survival and quality of life. There are also limited published guidelines about developing and running a multidisciplinary brain tumor clinic. Clinic personnel and policies vary between institutions, which limits the ability to study the effectiveness and optimal organization of brain tumor clinics. While considered as positive and effective by healthcare professionals, more research on multidisciplinary clinics is needed in order to optimize patient care and create standardized guidelines for the development of multidisciplinary brain tumor clinics in pediatrics. Research looking into patient/parent satisfaction with the multidisciplinary brain tumor clinic visits and care could provide information on how to best develop and run a multidisciplinary clinic. Also, researching outcomes of different clinics and institutions with different personnel involved in the multidisciplinary approach could give more information on which specialists are essential to multidisciplinary brain tumor clinics and which personnel could play an ancillary role. As multidisciplinary brain tumor clinics become more established, comparing patient outcomes and 
satisfaction between centers could help establish components and practices that ensure a successful clinic. As management and treatment continue to expand and improve for pediatric brain tumor patients, more personnel will continue to be involved in providing care for this group of patients and therefore the need for developing structured clinics to ensure that optimal patient care will always be the goal for centers treating patients with brain tumors.
Financial \& competing interests disclosure The authors have no relevant affiliations or financial involvement with any organization or entity with a financial interest in or financial conflict with the subject matter or materials discussed in the manuscript. This includes employment, consultancies, honoraria, stock ownership or options, expert testimony, grants or patents received or pending, or royalties.

No writing assistance was utilized in the production of this manuscript.

\section{References}

1 Pollack I. Pediatric brain tumors. Semin. Surg. Oncol. 16, 73-90 (1999)

2 Patel S, Bhatnagar A, Wear C et al. Are pediatric brain tumors on the rise in the USA? Significant incidence and survival findings from the SEER database analysis. Childs Nerv. Syst. 30, 147-154 (2014).

3 Wright FC, De Vito C, Langer B, Hunter A. Multidisciplinary cancer conference: a systemic review and development of practice standards. Eur. J. Cancer 43, 1002-1010 (2007).

4 Taylor C, Shewbridge A, Harris J, Green JS. Benefits of multidisciplinary teamwork in the management of breast cancer. Breast Cancer 5 , 79-85 (2013).

5 Look Hong NJ, Wright FC, Gagliardi AR, Paszat LF. Examining the potential relationship between multidisciplinary cancer care and patient survival: an international literature review. J. Surg. Oncol. 102, 125-134 (2010).

6 Petty JK, Vetto JT. Beyond doughnuts: tumor board recommendations influence patient care. J. Cancer Educ. 17(2), 97-100 (2002).

7 American Academy of Pediatrics. Standards for pediatric cancer centers. Pediatrics 134(2), 410-414 (2014).

8 European Partnership Action Against Cancer census group. Policy statement on multidisciplinary cancer care. Eur. J. Cancer 50, 475-480 (2014).

9 Sitarz R, Kocemba K, Maciejewski R, Polkowski WP. Effective cancer treatment by multidisciplinary teams. Polish J. Surg. 84(7), 371-376 (2012).

10 Kramer S, Meadows AT, Pastore G, Jarrett P, Bruce D. Influence of place of treatment on diagnosis, treatment, and survival in three pediatric solid tumors. J. Clin. Oncol. 2(8), 917-923 (1984).

11 Back MF, Ang E, Ng WH et al. Improvements in quality of care resulting from a formal multidisciplinary tumour clinic in the management of high-grade glioma. Ann. Acad. Med. Singapore 36(5), 347-351 (2007).

12 Do V, Gebski V, Barton MB. The effect of waiting for radiotherapy for grade III/IV glioma. Radiother. Oncol. 57, 131-136 (2000).

13 Smith ER, Butler WE, Barker II, FG. Craniotomy for resection of pediatric brain tumors in the United States, 1988-2000: effects of provider caseloads and progressive centralization and specialization of care. Neurosurgery 54, 553-565 (2004).

14 Huang T, Mueller S, Rutkowski MJ et al. Multidisciplinary care of patients with brain tumors. Surg. Oncol. Clin. N. Am. 22(2), 161-178 (2013).

15 Rosenblum ML, Mikkelsen T. Developing a brain tumor center. J. Neurooncol. 69, 169-180 (2004).

16 McMillan G. Narrative symposium: confronting pediatric brain tumors: parent stories. Narrat. Inq. Bioeth., 4(1), 1-3 (2014).

17 Schubart JR, Kinzie MB, Farace E. Caring for the brain tumor patient: family caregiver burden and unmet needs. Neuro Oncol. 10, 61-72 (2008).

18 Henrich N, Marra CA, Gastonguay L et al. De-escalation of therapy for pediatric medulloblastoma: trade-offs between quality of life and survival. Pediatr. Blood Cancer 61, 1300-1304 (2014).

19 Barr RD, Simpson T, Whitton A et al. Health-related quality of life in survivors of tumours of the central nervous system in childhood - a preference based approach to measurement in a cross sectional study. Eur. J. Cancer 35(2), 248-255 (1999).

20 Macartney G, VanDenKerkhof E, Harrison MB, Stacey D. Symptom experience and quality of life in pediatric brain tumor survivors: a cross-sectional study. J. Pain Sympt. Manag. 48(5), 957-967 (2014).
21 Klassen AF, Anthony SJ, Khan A, Sung L, Klaassen R. Identifying determinants of quality of life of children with cancer and childhood cancer survivors: a systematic review. Support. Care Cancer 19, 1275-1287 (2011).

22 Hocking MC, Hobbie WL, Deatrick JA et al. Neurocognitive and family functioning and quality of life among young adult survivors of childhood brain tumors. Clin. Neuropsychol. 25(6), 942-962 (2011).

23 Gurney JG, Kadan-Lottick NS, Packer RJ et al. Endocrine and cardiovascular late effects among adult survivors of childhood brain tumors. Cancer 97(3), 663-673 (2003).

24 Turner CD, Rey-Casserly C, Liptak CC, Chordas C. Late effects of therapy for pediatric brain tumor survivors. J. Child Neurol. 24(11), 1455-1463 (2009).

25 Brinkman TM, Delaney DL, Muriel AC et al. Suicide ideation in pediatric and adult survivors of childhood brain tumors. J. Neurooncol. 113, 425-432 (2013).

26 Blackmon JE, Liptak C, Recklitis CJ. Development and preliminary validation of a short form of the Beck Depression Inventory for Youth (BDY-I) in a sample of adolescent cancer survivors. J. Cancer Surviv. 9 (1), 107-114 (2015).

27 Bhat SR, Goodwin TL, Burwinkle TM et al. Profile of daily life in children with brain tumors: an assessment of health-related quality of life. J. Clin. Oncol. 23(24), 5493-5500 (2005).

28 Rummans TA, Clark MM, Sloan JA et al. Impacting quality of life for patients with advanced cancer with structured multidisciplinary intervention: a randomized control trial. J. Clin. Oncol. 24(4), 635-642 (2006).

29 Fuemmeler BF, Mullins LL, Marx BP. Posttraumatic stress and general distress among parents of children surviving a brain tumor. Child Health Care 30 (3), 169-182 (2001).

30 Yagci-Kupeli B, Akyuz C, Kupeli S, Buyukamukcu M. Health-related quality of 
life in pediatric cancer survivors: a multifactorial assessment including parental factors. J. Pediatr. Hematol. Oncol. 34(3), 194-199 (2012).

31 Matziou V, Perdikaris P, Feloni D et al. Cancer in childhood: children's and parent's aspects for quality of life. Eur. J. Oncol. 12, 209-216 (2008).

32 Landolt MA, Vollrath M, Niggli FK, Gnehm HE, Sennhouser FH. Health-related quality of life in children with newly diagnosed cancer: a one year follow-up study. Health Qual. Life Outcomes 4, 63 (2006).

33

Nelson RM, Botkin JR, Kodish ED et al. Palliative care for children. Pediatrics 106, 351-357 (2000).
34 Johnston DL, Nagal K, Friedman DL et al. Availability and use of palliative care and end-of-life services for pediatric oncology patients. J. Clin. Oncol. 26(28), 4646-4650 (2008).

35 Fraser LK, van Laar M, Miller M et al. Does referral to specialist paediatric palliative care services reduce hospital admissions in oncology patients at the end of life? $\mathrm{Br}$. J. Cancer 108, 1273-1279 (2013).

36 Chordas C, Manley P, Modest AM et al. Screening for pain in pediatric brain tumor survivors using the pain thermometer. J. Pediatr. Oncol. Nurs. 30(5), 249-259 (2013).

37 Lamb BW, Taylor C, Lamb LN et al. Facilitators and barriers to teamworking and patient centeredness in multidisciplinary cancer teams: findings of a national study. Ann. Surg. Oncol. 20, 1408-1416 (2013).

38 Jalil R, Ahmed M, Green JSA, Sevdalis N. Factors that can make an impact on decision making and decision implementation in cancer multidisciplinary teams: an interview study of the provider prospective. Int. J. Surg. 11, 389-394 (2013).

39 Ke KM, Blazeby JM, Strong S et al. Are multidisciplinary teams in secondary care cost-effective? A systematic review of the literature. Cost Eff. Resour. Alloc. 11, 7 (2013). 